PROCEEDINGS OF THE

AMERICAN MATHEMATICAL SOCIETY

Volurne 35, No. 1, September 1972

\title{
EUCLIDEAN NEIGHBORHOODS IN COMPACT SEMIGROUPS
}

JOHN D. MCCHAREN

\begin{abstract}
In this paper sufficient conditions are given under which a maximal idempotent in a compact connected semigroup cannot have a two-dimensional Euclidean neighborhood.
\end{abstract}

1. Introduction. It has been shown by Mostert and Shields [7] that the identity element of a compact connected semigroup cannot have a Euclidean neighborhood unless $S$ is a group. Later Cohen and Koch [1] proved that a right identity in $S$ cannot have a Euclidean neighborhood unless $S$ is left simple. These results are now corollaries to the fact that a right identity in a compact connected semigroup with a zero is peripheral [2, p. 168]. In this paper we use the concept of peripherality to obtain sufficient conditions under which maximal idempotents fail to have two-dimensional Euclidean neighborhoods. For an expository discussion of peripherality and its applications in this context, the reader is referred to [4].

2. Preliminary remarks. The notation of this paper is that of [8]. In particular $S$ will denote a topological semigroup, $K$ its minimal ideal, and $E$ is used to denote the set of idempotents. All spaces are assumed to be compact Hausdorff. The cohomology used is Alexander-WallaceSpanier theory with coefficient group arbitrary and reduced groups in dimension zero. If $A$ is a closed subset of a space $X$ and $h \in H^{n}(X)$, then $h \mid A$ denotes the image of $h$ under the natural homomorphism $H^{n}(X) \rightarrow$ $H^{\prime \prime}(A)$. We recall that a floor for an element $h$ of $H^{n}(X)$ is a closed subspace $F$ of $X$ such that $h \mid F \neq 0$ but $h \mid A=0$ for each proper closed subset $A$ of $F$. If $h \mid B \neq 0$ for some closed set $B$ in $X$, then a floor for $h$ exists and may be chosen as a subset of $B$. The following lemma gives sufficient conditions that a floor for a nonzero element $h$ of $H^{n}(X)$ be unique.

2.1 Lemma. Let $X$ be a space, $A$ and $B$ closed subsets of $X$ such that $X=A \cup B$ and $A \cap B=\{p\}$. If $h_{0} \in H^{n}(A)$ and $A$ is a floor for $h_{0}$, then there

Received by the editors April 15, 1971.

AMS 1970 subject classifications. Primary 22A15; Secondary 22A99.

Key words and phrases. Semigroups on continua, maximal idempotents.

(c) American Mathematical Society 1972 
exists an element $h$ in $H^{n}(X)$ such that (i) $h \mid A=h_{0}$, and (ii) if $C$ is a closed subset of $X$ such that $h \mid C \neq 0$, then $A \subset C$.

Proof. Consider the Mayer-Vietoris sequence

$\longrightarrow H^{n-1}(A \cap B) \longrightarrow H^{n}(X) \stackrel{J^{*}}{\rightarrow} H^{n}(A) \times H^{n}(B) \longrightarrow H^{n}(A \cap B) \longrightarrow$.

$J^{*}$ is onto so there exists $h \in H^{n}(X)$ such that $J^{*}(h)=\left(h_{0}, 0\right)$; i.e. $h \mid A=h_{0}$ and $h \mid B=0$. Suppose $C$ is a closed subset of $X$ such that $h \mid C \neq 0$ and consider the exact sequence

$$
\longrightarrow H^{n-1}(A \cap B \cap C) \longrightarrow H^{n}(C) \stackrel{J^{*}}{\longrightarrow} H^{n}(A \cap C) \times H^{n}(B \cap C) \longrightarrow .
$$

Since $J^{*}$ is $1-1, J^{*}(h \mid C) \neq 0$. Since $h \mid B \cap C=0$, we have $h \mid A \cap C \neq 0$. But $A$ is a floor for $h_{0}$ and $h\left|A \cap C=h_{0}\right| A \cap C$, so $A \cap C=A$. This establishes the conclusion of the theorem.

A point $p$ in a space $X$ is peripheral if there exist small open sets $V$ containing $p$ such that the induced mapping $H^{n}(X) \rightarrow H^{n}(X \backslash V)$ is an isomorphism for all $n \geqq 0$. That is to say there exist small open sets containing $p$ such that $H^{n}(X, X \backslash V)=0$ for all $n \geqq 0$. By the map excision theorem it follows that $p$ is peripheral if and only if $H^{n}\left(V^{*}, V^{*} \backslash V\right)=0$ for all $n \geqq 0$. The local nature of the concept of peripherality is thus clearly seen; i.e. if $A$ is a closed subset of a space $X$ and $p \in A^{\circ}$, then $p$ is peripheral in $A$ if and only if $p$ is peripheral in $X$. These remarks together with the aforementioned result concerning a right identity in a semigroup yield the following lemma.

2.2 Lemma. Let $S$ be a compact connected semigroup with a zero and suppose $e$ and $f$ are idempotents in $S$ such that $S=S e \cup S f$. Then either $e \in S f$, in which case $S=S f$, or e is peripheral in $S$.

The following theorem is proved in [5] and is of importance to the sequel.

2.3 Theorem. If $S$ is a compact connected semigroup with a zero satisfying $S=E S E$, and $h$ is a nonzero element of $H^{2}(S)$, then there exists a pair of idempotents $e$ and $f$ such that $h \mid S e \cup S f \neq 0$.

Recall that the $\leqq(\mathscr{L}) \& \leqq(\mathscr{J})$ orderings in a semigroup $S$ are defined as follows: for $x, y \in S$, (i) $x \leqq(\mathscr{L}) y$ if $x \cup S x \subset y \cup S y$, and (ii) $x \leqq(\mathscr{J}) y$ if $x \cup S x \cup x S \cup S x S \subset y \cup S y \cup y S \cup S_{y} S$. We say that an element $x$ in $S$ is $\mathscr{L}$-maximal $(\mathscr{J}$-maximal) if $x$ is maximal relative to the ordering $\leqq \mathscr{L}$ $(\leqq \mathscr{J})$. It is known that a $\mathscr{J}$-maximal idempotent is $\mathscr{L}$-maximal in a compact semigroup. This together with the preceding remarks yield the following corollary to Lemma 2.2. 
2.4 Corollary. If $S$ is a continuum with a zero, $S=E S E$, and $e$ is a $\mathscr{J}$-maximal idempotent of $S$, then e does not belong to the unique floor of any nonzero element of $H^{2}(S)$.

Proof. Suppose that $h \in H^{2}(S)$ and $F$ is the unique floor for $h$. First one should observe that no point of $F$ is peripheral in any closed subset $A$ of $S$ which contains $F$. Indeed if $p \in F$ and $V$ is any $A$-open set containing $p$, then $h \mid A \backslash V=0$ since $F$ is the unique floor for $h$. Therefore the natural homomorphism $H^{n}(A) \rightarrow H^{n}(A \backslash V)$ is not $1-1$ and so $p$ is not peripheral in $A$.

Now by Theorem 2.3 there exist idempotents $g$ and $f$ in $S$ such that $h \mid S g \cup S f \neq 0$, and therefore $F \subset S g \cup S f$. Thus, if $e$ is a $\mathscr{J}$-maximal idemptoent and $e \in F$, then it may be assumed that $e \in S g$. Since $e$ is also $\mathscr{L}$-maximal it follows that $S e=S g$, so $F \subset S e \cup S f$. Because $h \mid S f=0$ and $h \mid S e \cup S f \neq 0$ it must be that $e \notin S f$, so by Lemma 2.2 , $e$ is peripheral in $S e \cup S f$. This contradicts the above and the proof is complete.

The following lemma concerns the topology of the plane and a proof is only sketched here.

2.5 Lemma. Let $\boldsymbol{R}^{2}$ denote the Euclidean plane. Suppose $N$ is a closed totally disconnected set in $\boldsymbol{R}^{2}$ containing the distinguished point p. Then there exists a compact neighborhood $M$ of p satisfying: (i) $F(M) \cap N=\square$, (ii) $F(M)$ is connected, (iii) if $C$ is any closed connected subset of $M$ containing $F(M)$ and $D$ is any component of $M \backslash C$, then $D$ is homeomorphic to the open unit disk.

Proof. Let $\Omega$ be the point at infinity. Applying Theorem 20 of R. L. Moore's Chapter IV to the points $p$ and $\Omega$ of the closed and totally disconnected subset $N \cup\{\Omega\}$ of the sphere $\boldsymbol{R}^{2} \cup\{\Omega\}$. one obtains a simple closed curve $J$ such that $p$ is in the interior of $J$ and $J \cap(N \cup\{\Omega\})=\varnothing$. Letting $M=J$ and its interior, claims (i), (ii) and (iii) follow immediately.

3. Principal results. We proceed to give sufficient conditions under which maximal idempotents in compact semigroups fail to have twodimensional Euclidean neighborhoods. The motivation is to give conditions under which we may find an ideal $J$ in $S$ such that in the Rees quotient $S / J$, e lies in a 2-sphere neighborhood; thus establishing a contradiction to Corollary 2.4. We give the main theorem now.

3.1 THEOREM. Let $S$ be a continum satisfying $S=E S E$. If $e$ is a $\mathscr{J}$-maximal idempotent of $S$ and $\mathscr{J} e$ is totally disconnected, then $e$ does not lie in a two-dimensional Euclidean neighborhood.

Proof. Suppose $e$ is an element of a two-dimensional neighborhood $V$. Since $\mathscr{J} e$ is totally disconnected, $e \notin K$. Let $M$ be a neighborhood of 
$e$ satisfying the conclusion of Lemma 2.5. (Here $N$ is taken to be $\mathscr{J}_{e} \cap V$ and $e$ the distinguished point.) It is well known that $S \backslash \mathscr{J}_{e}$ is a maximal ideal of $S$, and since $F(M) \cap \mathscr{J}_{e}=\square$, the ideal $S F(M) S$ is contained in $S \backslash \mathscr{J}_{e}$. Moreover $F(M) \subset S F(M) S$ since $S=E S E$, and because $F(M)$ is connected, the set $C=S F(M) S \cap M$ is connected. Letting $D$ denote the component of $M \backslash C$ containing $e$, we have that $D$ is homeomorphic to the open unit disk of $\boldsymbol{R}^{2}$.

Let $J=S F(M) S$, and consider the Rees quotient modulo $J$ with natural map $p: S \rightarrow S / J$. Clearly $p(D)$ is homeomorphic to $D$ and it is easily verified that $p(D)$ is open in $S / J$, and $p(D)^{*} \mid p(D)=p(J)$; i.e. $p(D)$ is homeomorphic to an open disk and its boundary is a point. Therefore $p(D)^{*}$ is homeomorphic to a 2-sphere. The hypotheses of Lemma 2.1 are satisfied so $p(D)^{*}$ is the unique floor for some element $h$ in $H^{2}(p(S))$. The fact that $p(e)$ is a maximal idempotent in $S / J$, together with the preceding statement, yields a contradiction to Corollary 2.4. Therefore $e$ does not have a twodimensional Euclidean neighborhood.

There are several instances where a maximal idempotent $e$ fails to have a two-dimensional Euclidean neighborhood. In particular, let $S$ be a continuum satisfying $S^{2}=S$ and $S \neq K$. If a maximal idempotent $e$ has a two-dimensional Euclidean neighborhood and $H_{e}$ is totally disconnected then so is $\mathscr{J}_{e}$. If in addition to the above, $S=S e S$, then $H_{e}$ is necessarily totally disconnected [6]. We thus obtain the following corollaries:

3.2 Corollary. Let $S$ be a continuum with $S=E S E$, and suppose e is a maximal idempotent in $S$. If $\mathscr{H}_{e}$ is totally disconnected and e has a twodimensional Euclidean neighborhood, then $S=K$.

3.3 Corollary. Let $S$ be a continuum with $S=E S E$ and $S=S e S$ for some (maximal) idempotent $e$ in $S$. If $e$ has a two-dimensional Euclidean neighborhood, then $S=K$.

3.4 COROLIARY. Let $S$ be a continuum satisfying $S=E S E$, and suppose $e$ is a $\mathscr{J}$-maximal idempotent of $S$. If $S$ is a subset of the plane $\boldsymbol{R}^{2}$ and $S \neq K$, then $e$ lies on the boundary of $S$ in $\boldsymbol{R}^{2}$.

Proof. If $\mathscr{H}_{*}$ is totally disconnected, the conclusion follows from Corollary 3.2. Otherwise $e$ lies on a circle subgroup $G_{0}$ of $H_{e}$ which separates $\boldsymbol{R}^{2}$. But $G_{0}$ does not separate $S$ [3], so the conclusion follows.

\section{BIBLIOGRAPHY}

1. H. Cohen and R. J. Koch, Acyclic semigroups and multiplications on two-manifolds, Trans. Amer. Math. Soc. 118 (1965), 420-427. MR 30 \#5283.

2. K. H. Hofmann and P. S. Mostert, Elements of compact semigroups, Merrill, Columbus, Ohio, 1966. MR 35 \#285. 
3. R. J. Koch and A. D. Wallace, Maximal ideals in compact semigroups, Duke Math. J. 21 (1954), 681-685. MR 16, 112.

4. — Compact connected spaces supporting topological semigroups, Semigroup Forum 1 (1971), no. 2, 95-102. MR 42 \#3220.

5. John D. McCharen, Semigroups on finitely floored spaces, Trans. Amer. Math. Soc. 156 (1971), 85-89. MR 43 \#404.

6. - Dissertation, Louisiana State University, Baton Rouge, La., 1969.

7. P. S. Mostert and A. L. Shields, Semigroups with identity on a manifold, Trans. Amer. Math. Soc. 91 (1959), 380-389. MR 21 \#4204.

8. A. D. Wallace, The structure of topological semigroups, Bull. Amer. Math. Soc. 61 (1955), 95-112. MR 16, 796.

Department of Mathematics, Northern Illinois University, DeKalb, Illinois 60115 